\title{
Stochastic uncertainty models for the luminance consistency assumption
}

\author{
Thomas Corpetti ${ }^{1}$ and Etienne Mémin ${ }^{2}$ \\ 1 : CNRS/LIAMA, Institute of Automation, PO Box 2728, Beijing 100190, PR China \\ 2 : INRIA, Campus Universitaire de Beaulieu, 35042 Rennes Cedex, France \\ tcorpetti@gmail.com, memin@inria.fr
}

\begin{abstract}
In this paper, a stochastic formulation of the brightness consistency used in many computer vision problems involving dynamic scenes (motion estimation or point tracking for instance) is proposed. Usually, this model which assumes that the luminance of a point is constant along its trajectory is expressed in a differential form through the total derivative of the luminance function. This differential equation links linearly the point velocity to the spatial and temporal gradients of the luminance function. However when dealing with images, the available informations only hold at discrete time and on a discrete grid. In this paper we formalize the image luminance as a continuous function transported by a flow known only up to some uncertainties related to such a discretization process. Relying on stochastic calculus, we define a formulation of the luminance function preservation in which these uncertainties are taken into account. From such a framework, it can be shown that the usual deterministic optical flow constraint equation corresponds to our stochastic evolution under some strong constraints. These constraints can be relaxed by imposing a weaker temporal assumption on the luminance function and also in introducing anisotropic intensity-based uncertainties. We in addition show that these uncertainties can be computed at each point of the image grid from the image data and provide hence meaningful information on the reliability of the motion estimates.

To demonstrate the benefit of such a stochastic formulation of the brightness consistency assumption, we have considered a local least squares motion estimator relying on this new constraint. This new motion estimator improves significantly the quality of the results.
\end{abstract}

\section{INTRODUCTION}

Many computer vision problems are formulated on the basis of the spatial and temporal variations of the image luminance. For instance in all approaches requiring the tracking along time of points, curves or surfaces [32], the evolution of the luminance function provides a crucial information that can be used as a dynamic constraint [27], [28] or as an observation measurement [17], [22], [30]. In several domains where physical flows are involved (meteorology, oceanography), such an evolution law describes the transportation of a physical scalar data, $f$, by a motion field, $v$, under a global conservation constraint using the classical relation:

$$
\frac{d f}{d t}=\frac{\partial f}{\partial t}+\boldsymbol{v} \cdot \nabla f=0,
$$

where $\boldsymbol{\nabla}$ is the gradient operator in the $x$ and $y$ directions. When the function $f$ denotes the luminance function this equation is referred in computer vision as the Optical Flow Constraint Equation (OFCE) or as the brightness consistency assumption and constitutes the only available information for motion estimation issues. Optical flow estimation has been studied intensively since the seminal work of Horn and Schunck [16] and a huge number of methods based on diverse variations of this constraint have been proposed in the literature [7], [12], [31], [33], [34]. Usually a data model constructed from this constraint is associated with some spatial regularizers that promote motion fields with some spatial (and sometimes temporal) coherency. Many authors have proposed on this basis very efficient techniques. Readers can refer to [5]-[8], [19], [23]-[25], [29], [35], [36], [38] for a non exhaustive panel or [15] for a recent review on estimators dedicated to fluid flows. Comparative performance evaluations of some of these techniques can be found in [2], [3], [13], [15]. Among the developed approaches, the techniques focused first on the design of new regularization terms (able for instance to deal with occlusions, discontinuities or relying on physical grounds [11], [15]) and second on the application of advanced minimization strategies. Surprisingly, apart for some specific applications devoted to some specific types of imagery (fluid, biology, infrared imagery, tomography, IRM, ...), only very few authors have worked on generic alternative data terms to the classical brightness consistency assumption, despite the fact it plays a crucial role in the motion estimation process.

Besides, the motion estimation issue should be seen in its most accomplished goal as a velocity or deformation metrology problem in which one aims at recovering accurate motion measurements and not only as a technique providing approximate estimate of velocity vectors that inevitably give rise to erroneous drifts when integrated along time. Such drifts can be efficiently attenuated considering motion tracking procedures based on stochastic filtering [1] or optimal control [27]. These temporal integration approaches require the introduction of uncertainty models quantifying the reliability of the measurements. An accurate modeling of these uncertainties is crucial as a large uncertainty values favors forecast motion values provided by the state variable dynamics whereas small uncertainty values encourages a high confidence on the measurements. In this study 
we aim at proposing a framework that allows us estimating local motion measurements but also their uncertainties. To our knowledge such uncertainty measurements are provided by none of the existing motion estimation techniques.

The conventional optical flow constraint relation (1) is in fact defined as the differential of a function known only on spatial and temporal discrete point positions (related to the image sequence spatio-temporal lattice). This is somewhat a strong constraint since in practice, the grid points on which is defined the luminance is transported by a flow itself known only up to the same discrete positions. It results from this discretization process an inherent uncertainty on the points location that can reveal to be of important magnitude when are involved strong motions, large inter frames lapse rate or crude spatial discretization associated for instance to large spatial scales measurements. The idea is therefore to encode such a location uncertainty as a random variable and to incorporate the uncertainty transportation into the brightness consistency assumption. Stochastic calculus provides the differentiation rules needed to formalize such evolution law of uncertainty terms. In this paper isotropic or anisotropic models of uncertainty have been settled yielding thus to two different versions of a brightness assumption data model under location uncertainty. Let us point out that such a location uncertainty modeling allows us proposing a natural continuous multiscale estimation associated to a hierarchical discretization process on nested discrete latices. Uncertainty models parameters are seen as additional parameters that have to be jointly estimated within the motion estimation procedure. The overall resulting scheme provides a proper way to measure in a joint way motion vectors and their associated uncertainty from the data. Our new models enable to provide

1) a more accurate consistency conservation assumption equation that can be used in many computer vision problems,

2) an estimate of the motion uncertainties, and

3) a natural continuous multiscale strategy for the motion estimation strategy.

We have the conviction that the association of an uncertainty measure to the variable estimated is of high importance in many applications. For instance in the context of motion estimation, this uncertainty is directly related to the accuracy of the velocity field. For tracking issue, such uncertainties are also linked to the confidence one may have on the conservation of any scalar field defined on the image grid. For problem in which one aims at forecasting these scalar fields the uncertainties enable to simulate various configurations of the displaced scalar field image. This is may have many practical applications (in geosciences in particular) when one wishes to predict scalar transported by the flow such as temperature, pollutant sheets, or micro-organisms density.

In this paper in order to validate our new data terms, we have designed a simple local motion estimator based on the principles of the Lucas-Kanade estimator [21]. We have used our new stochastic relations of the image luminance as an observation operator and compared it with the standard optical flow constraint (1). In addition, an original multiscale scheme also interpreted as a stochastic uncertainty on the pixel grid is presented and evaluated. Experiments are carried out on fluid particles images and on images of the Middleburry database. In the context of particles images, local approaches are currently used in operational systems. Those local techniques based on correlation have shown to be very efficient in this context. On particle images, dense motion estimation techniques may reach the same level of accuracy with an greater density of measurements when regularization terms reflecting well the physical properties of the fluid are considered [10], [11], [14], [39]. As for the second experiments, on several images issued from the Middleburry dataset, we analyze the benefit of a data term based on the stochastic models proposed in this paper compared to a classic evolution based on (1). The experimentations indeed reveal a better accuracy of the motion fields provided by our models, and we also highlight the benefit of the uncertainty maps extracted. Lastly we present the evaluation on the complete Middleburry database.

The paper is organized as follows: in section II we define a stochastic version of the luminance function, by incorporating isotropic and anisotropic uncertainties. From this formulation, two conservation constraints of the image luminance are derived. If the velocity field is available or if we estimate it simultaneously, we propose in section III a way to compute the associated uncertainty. Finally, section IV presents a local multiscale Lucas and Kanade motion estimator based on the brightness consistency stochastic models.

\section{STOCHASTIC LUMINANCE FUNCTION AND CONSERVATION CONSTRAINTS}

\section{A. Notations - Conventions}

In this paper we use the following conventions/notations:

- the image luminance is $f$;

- we represent as a vector $\boldsymbol{X}=\left(\boldsymbol{X}^{1}, \ldots, \boldsymbol{X}^{m}\right)^{T}$ a grid of $2 D$ points, $\boldsymbol{X}^{s} \in \mathbb{R}^{2}$;

- the "pixel" grid of the images $\boldsymbol{X}_{t-1}$ is represented by the position of a grid $\boldsymbol{X}$ at the initial time, set to $t-1$ (see figure $1(\mathrm{a}))$

- at time $t-1$, this grid is driven by a velocity field $\boldsymbol{v}\left(\boldsymbol{X}_{t-1}, t-1\right): \mathbb{R}^{2 m} \times \mathbb{R}^{+} \rightarrow \mathbb{R}^{2 m}$ defined on the initial grid $\boldsymbol{X}_{t-1}$ to generate the new point positions $\boldsymbol{X}_{t}$ at time $t$ (see figure 1 (b)).

\section{B. Stochastic luminance function}

We first write the image luminance as the function of a stochastic process related to the position of image points. If one assumes, as illustrated in figure 1, that the velocity $\boldsymbol{v}$ to estimate transports the grid from $\boldsymbol{X}_{t-1}$ to $\boldsymbol{X}_{t}$ up to a Brownian 


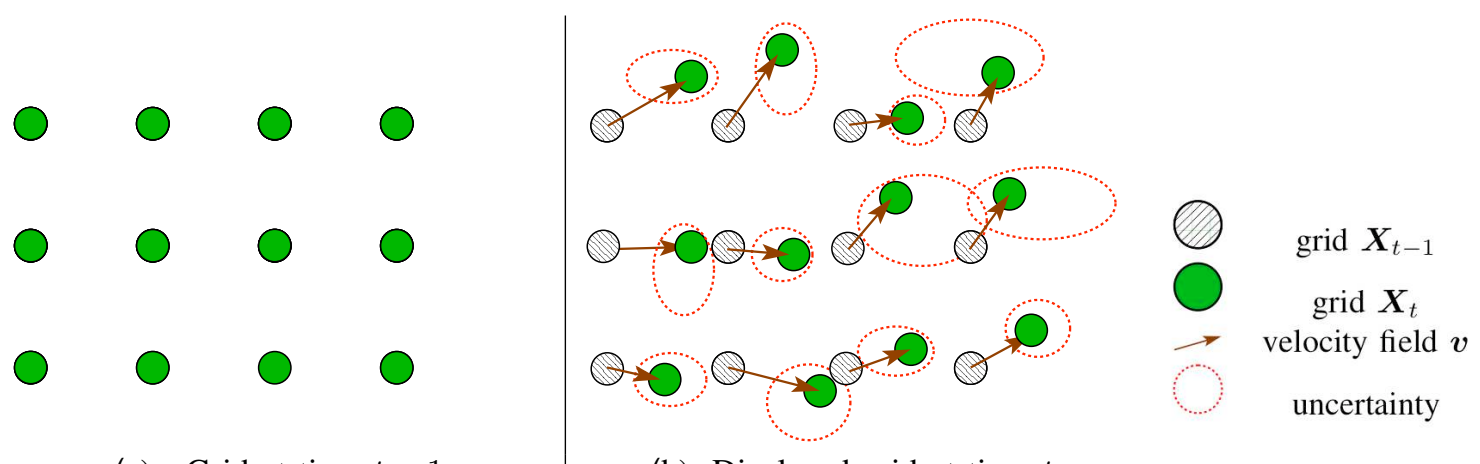

(a) : Grid at time $t-1$

(b): Displaced grid at time $t$

Fig. 1. Displacement of the grid of points. The initial grid at time $t-1$ in (a) is transported by the velocity field $\boldsymbol{v}$ to reach the configuration at time $t$ represented in (b), up to some uncertainties (dashed lines).

motion, we can write:

$$
d \boldsymbol{X}_{t}=\boldsymbol{v}\left(\boldsymbol{X}_{t-1}, t-1\right) d t+\boldsymbol{\Sigma}\left(t, \boldsymbol{X}_{t}\right) d \mathbf{B}_{t},
$$

where $\mathbf{B}_{t}=\left(\mathbf{B}_{t}^{1}, \ldots, \mathbf{B}_{t}^{m}\right)^{T}$ is a multidimensional standard Brownian motion of $\mathbb{R}^{2 m}, \boldsymbol{\Sigma}$ a $(2 m \times 2 m)$ covariance matrix and $d \boldsymbol{X}_{t}=\boldsymbol{X}_{t}-\boldsymbol{X}_{t-1}$ represents the difference between the grid positions. The luminance function $f$ usually defined on spatial points $\boldsymbol{x}=(x, y)$ at time $t$ is here defined on the grid as a map from $\mathbb{R}^{+} \times \mathbb{R}^{2 m}$ into $\mathbb{R}^{m}$ and is assumed to be $C^{1,2}\left(\mathbb{R}^{+}, \mathbb{R}^{2 m}\right)$. Its differential is obtained following the differentiation rules of stochastic calculus (the so called Itô formulae) that gives the expression of the differential of any continuous function of an Itô diffusion of the form 2 (see [26] for an introduction to stochastic calculus):

$$
\begin{aligned}
d f\left(\boldsymbol{X}_{t}, t\right)= & \frac{\partial f}{\partial t} d t+\sum_{i=(1,2)} \frac{\partial f\left(\boldsymbol{X}_{t}, t\right)}{\partial x_{i}} d X_{t}^{i}+ \\
& \frac{1}{2} \sum_{(i, j)=(1,2) \times(1,2)} \frac{\partial^{2} f\left(\boldsymbol{X}_{t}, t\right)}{\partial x_{i} \partial x_{j}} d<X_{t}^{i}, X_{t}^{j}>.
\end{aligned}
$$

The term $<X_{t}^{i}, X_{t}^{j}>$ denotes the joint quadratic variations of $X^{i}$ and $X^{j}$ defined as the limit in probability:

$$
<X_{t}^{i}, X_{t}^{j}>=\lim _{\delta t_{k} \rightarrow 0}^{\mathbb{P}} \sum_{t_{k} \leq t}\left(X_{t_{k-1}}^{i}-X_{t_{k}}^{i}\right)\left(X_{t_{k-1}}^{j}-X_{t_{k}}^{j}\right),
$$

which can be computed according to the following rules:

$$
\begin{aligned}
& <B^{i}, B^{j}>=\delta_{i j} t \\
& <h(t), h(t)>=<h(t), d B^{i}>=<B^{j}, h(t)>=0,
\end{aligned}
$$

where $\delta_{i j}=1$ if $i=j, \delta_{i j}=0$ otherwise, and $h(t)$ is a deterministic function. Compared to classical differential calculus, new terms related to the Brownian random terms have been introduced in this stochastic formulation. A possible way to represent the stochastic part of (2) is to use an isotropic uncertainty variance map $\sigma\left(X_{t}, t\right): \mathbb{R}^{+} \times \mathbb{R}^{2 m} \rightarrow \mathbb{R}^{m}$

$$
\boldsymbol{\Sigma}\left(\boldsymbol{X}_{t}, t\right) d \mathbf{B}_{t}=\operatorname{diag}\left(\sigma\left(\boldsymbol{X}_{t}, t\right)\right) \otimes \mathbb{I}_{2} d \mathbf{B}_{t},
$$

where $\mathbb{I}_{2}$ is the $(2 \times 2)$ identity matrix, and $\otimes$ denotes the Kronecker product. Alternatively, one can use anisotropic intensitybased uncertainties along the normal (with a variance $\sigma_{\eta}$ ) and the tangent (with a variance $\sigma_{\tau}$ ) of the photometric contour following:

$$
\boldsymbol{\Sigma}\left(\boldsymbol{X}_{t}, t\right) d \mathbf{B}_{t}=\operatorname{diag}\left(\sigma_{\eta}\left(\boldsymbol{X}_{t}, t\right)\right) \otimes \boldsymbol{\eta} d B_{t}^{\eta}+\operatorname{diag}\left(\sigma_{\tau}\left(\boldsymbol{X}_{t}, t\right)\right) \otimes \boldsymbol{\tau} d B_{t}^{\tau} .
$$

The vectors

$$
\boldsymbol{\eta}=\frac{1}{|\boldsymbol{\nabla} f|}\left(\begin{array}{c}
f_{x} \\
f_{y}
\end{array}\right), \boldsymbol{\tau}=\frac{1}{|\boldsymbol{\nabla} f|}\left(\begin{array}{c}
-f_{y} \\
f_{x}
\end{array}\right),
$$

represent respectively the normal and tangent of the photometric isolines, $B^{\eta}$ and $B^{\tau}$ are two scalar independant multidimensional Brownian noises of $\mathbb{R}^{m}$ and $f_{\bullet}=\partial f\left(X_{t}, t\right) / \partial \bullet$ for $\bullet=(x, y)$. Let us now express the luminance variations $d f\left(X_{t}, t\right)$ under such isotropic or anisotropic uncertainties.

1) Isotropic uncertainties: Applying Itô formula (3) to the isotropic uncertainty model yields a luminance variation defined as:

$$
d f\left(\boldsymbol{X}_{t}, t\right)=\left(\frac{\partial f}{\partial t}+\nabla f \cdot \boldsymbol{v}+\frac{1}{2} \sigma^{2} \Delta f\right) d t+\sigma \nabla f \cdot d \boldsymbol{B}_{t} .
$$


2) Anisotropic uncertainties: Considering the anisotropic uncertainty model (6), the corresponding quadratic variations read:

$$
\begin{aligned}
& d<\boldsymbol{X}_{t}^{1}, \boldsymbol{X}_{t}^{1}>=\frac{1}{|\nabla f|^{2}}\left(\sigma_{\eta}^{2} f_{x}^{2}+\sigma_{\tau}^{2} f_{y}^{2}\right) d t \\
& d<\boldsymbol{X}_{t}^{2}, \boldsymbol{X}_{t}^{2}>=\frac{1}{|\nabla f|^{2}}\left(\sigma_{\eta}^{2} f_{y}^{2}+\sigma_{\tau}^{2} f_{x}^{2}\right) d t \\
& d<\boldsymbol{X}_{t}^{1}, \boldsymbol{X}_{t}^{2}>=\frac{1}{|\nabla f|^{2}}\left(f_{x} f_{y}\right)\left(\sigma_{\eta}^{2}-\sigma_{\tau}^{2}\right) d t
\end{aligned}
$$

and the variation of luminance, $d f$, reads now:

$$
\begin{aligned}
d f\left(\boldsymbol{X}_{t}, t\right)= & \left(\frac{\partial f}{\partial t}+\boldsymbol{\nabla} f \cdot \boldsymbol{v}+\frac{\boldsymbol{\nabla} f^{T} \boldsymbol{\nabla}^{2} f \boldsymbol{\nabla} f}{2|\boldsymbol{\nabla} f|^{2}}\left(\sigma_{\eta}^{2}-\sigma_{\tau}^{2}\right)\right. \\
& \left.+\frac{\sigma_{\tau}^{2} \Delta f}{2}\right) d t+\sigma_{\eta}\|\boldsymbol{\nabla} f\| d B_{t}^{\eta}+\underbrace{\sigma_{\tau} \boldsymbol{\nabla} f^{T} \boldsymbol{\tau} d B_{t}^{\tau}}_{=0} .
\end{aligned}
$$

In this brightness variation model the stochastic term related to the uncertainty along the tangent vanishes (since the projection of the gradient along the level lines is null).

It is straightforward to remark that the standard brightness consistency assumption is obtained from (7) or (11) using zero uncertainties $\left(\sigma=\sigma_{\eta}=\sigma_{\tau}=0\right.$ ). The proposed stochastic formulation enables thus to use a softer constraint. From this formulation, let us now derive generic models for the evolution of the image luminance transported by a velocity fields with location uncertainty.

\section{Uncertainty models for luminance conservation}

Starting from a known grid $\boldsymbol{X}_{t-1}$ and its corresponding velocity, the conservation of the image luminance can be quite naturally expressed from the conditional expectation $\mathbb{E}\left(d f\left(\boldsymbol{X}_{t}, t\right) \mid \boldsymbol{X}_{t-1}\right)$ between $t-1$ and $t$. To compute this term, we exploit the fact (as shown in appendix A) that the expectation of any function $\Psi\left(\boldsymbol{X}_{t}, t\right)$ of a stochastic process $d \boldsymbol{X}_{t}$ (as in (2)) knowing the grid $\boldsymbol{X}_{t-1}$ reads:

$$
\mathbb{E}\left(\Psi\left(\boldsymbol{X}_{t}, t\right) \mid \boldsymbol{X}_{t-1}\right)=\Psi\left(\boldsymbol{X}_{t-1}+\boldsymbol{v}, t\right) * \mathcal{N}(0, \boldsymbol{\Sigma}),
$$

where $\mathcal{N}(0, \boldsymbol{\Sigma})$ is a multidimensional centered Gaussian. This latter relation indicates that the expectation of a function $\Psi\left(\boldsymbol{X}_{t}, t\right)$ knowing the location $\boldsymbol{X}_{t-1}$ under a Brownian uncertainty of variance $\boldsymbol{\Sigma}$ is obtained by a convolution of $\Psi\left(\boldsymbol{X}_{t-1}+\boldsymbol{v}, t\right)$ with a centered Gaussian kernel of variance $\boldsymbol{\Sigma}$.

Assuming $\boldsymbol{\Sigma}$ known, our new luminance variation model $\mathbb{E}\left(d f\left(\boldsymbol{X}_{t}, t\right) \mid \boldsymbol{X}_{t-1}\right)$ is hence defined as:

$$
\begin{aligned}
\mathbb{E}\left(d f\left(\boldsymbol{X}_{t}, t\right) \mid \boldsymbol{X}_{t-1}\right) & =g_{\boldsymbol{\Sigma}} *\left(d f\left(\boldsymbol{X}_{t-1}+\boldsymbol{v}, t\right)\right) \\
& =g_{\boldsymbol{\Sigma}} * \mathcal{H}(f, \boldsymbol{v}) d t
\end{aligned}
$$

where the function $\mathcal{H}(f, \boldsymbol{v})$ corresponds to the bounded variation part of the luminance differential. Its form depends on the type of uncertainty considered. For an isotropic diffusion, the expectation of this function reads

$$
\mathbb{E}\left(\mathcal{H}(f, \boldsymbol{v}) \mid \boldsymbol{X}_{t-1}\right)=g_{\sigma} *(\boldsymbol{\nabla} f \cdot \boldsymbol{v}+\frac{\partial f}{\partial t}+\underbrace{\frac{1}{2} \sigma^{2} \Delta f}_{\mathcal{F}(f)}),
$$

whereas for the anisotropic version it is:

$$
\begin{aligned}
& \mathbb{E}\left(\mathcal{H}(f, \boldsymbol{v}) \mid \boldsymbol{X}_{t-1}\right)=g_{\boldsymbol{\Sigma}^{*}} \\
& (\boldsymbol{\nabla} f \cdot \boldsymbol{v}+\frac{\partial f}{\partial t}+\underbrace{\frac{\boldsymbol{\nabla} f^{T} \boldsymbol{\nabla}^{2} f \boldsymbol{\nabla} f}{2|\boldsymbol{\nabla} f|^{2}}\left(\sigma_{\eta}^{2}-\sigma_{\tau}^{2}\right)+\frac{\sigma_{\tau}^{2} \Delta f}{2}}_{\mathcal{F}(f)}) .
\end{aligned}
$$

If the brightness conservation constraint strictly holds, one obtains $\sigma=\sigma_{\eta}=\sigma_{\tau}=0$; the Gaussian kernels turn to Dirac distributions and relations (13), (14) and (15) correspond to (1). The proposed model brings thus a natural extension of the usual brightness consistency data model. Furthermore as a bonded variation process $\mathcal{H}(f, \boldsymbol{v})$ has a null quadratic variation:

$$
\mathbb{E}\left(\mathcal{H}^{2}(f, \boldsymbol{v})\right)=g_{\boldsymbol{\Sigma}} * \mathcal{H}^{2}(f, \boldsymbol{v})=0
$$

An approximation of this relation for a locally constant velocity in space will provide us a local least squares estimation scheme for the unknown velocity. Before presenting this procedure, in the next section we propose a way to estimate the uncertainties $\sigma_{\eta}$ and $\sigma_{\tau}$. 


\section{UNCERTAINTY ESTIMATION}

Assuming an observed motion field $\boldsymbol{v}_{\boldsymbol{o b s}}$ that transports the luminance is available (we will describe in section IV a local technique for this estimation), it is possible to estimate the uncertainties $\sigma_{\eta}(\boldsymbol{x}, t)$ and $\sigma_{\tau}(\boldsymbol{x}, t)$ for each location $\boldsymbol{x}$ at time $t$.

\section{A. Estimation of $\sigma_{\eta}$}

Computing the quadratic variation of the luminance function $d f$ between $t-1$ and $t$ using the properties in (4) yields, for the isotropic or anisotropic version:

$$
\left.d\left\langle f\left(\boldsymbol{X}_{t}, t\right), f\left(\boldsymbol{X}_{t}, t\right)\right\rangle=\sigma_{\eta}^{2}\left(\boldsymbol{X}_{t}, t\right) \| \nabla f\left(\boldsymbol{X}_{t}, t\right)\right) \|^{2},
$$

where $\sigma=\sigma_{\eta}$ in the isotropic formulation. This quadratic variation can also be approximated from the luminance $f$ by:

$$
d\left\langle f\left(\boldsymbol{X}_{t}, t\right), f\left(\boldsymbol{X}_{t}, t\right)\right\rangle \approx\left(f\left(\boldsymbol{X}_{t}, t\right)-f\left(\boldsymbol{X}_{t-1}, t-1\right)\right)^{2} .
$$

As convergence in probability implies convergence in distribution, the conditional expectation of both previous terms should be identical, and one can estimate the variance by:

$$
\sigma_{\eta}\left(\boldsymbol{X}_{t}\right)=\sqrt{\frac{\mathbb{E}\left(f\left(\boldsymbol{X}_{t}, t\right)-f\left(\boldsymbol{X}_{t-1}, t-1\right)\right)^{2}}{\left.\mathbb{E}\left(\| \boldsymbol{\nabla} f\left(\boldsymbol{X}_{t}, t\right)\right) \|^{2}\right)}} .
$$

The expectation in the numerator and denominator are then computed at the displaced point $X_{t-1}+\boldsymbol{v}_{\text {obs }}\left(\boldsymbol{X}_{t-1}\right)$ through the convolution of variance $\boldsymbol{\Sigma}\left(\boldsymbol{X}_{t-1}, t-1\right)$. A recursive estimation process is thus emerging from equation (19). Let us note this recursion corresponds to the following least squares estimation:

$$
\left.\sigma_{\eta}^{2}=\arg \min _{\sigma}\left[\mathbb{E} d f^{2}\left(\boldsymbol{X}_{t}, t\right)-\sigma^{2} \mathbb{E} \| \nabla f\left(\boldsymbol{X}_{t}, t\right)\right) \|^{2}\right]^{2},
$$

where $d f^{2}\left(\boldsymbol{X}_{t}, t\right)$ is a shortcut notation for the quadratic variation $d\left\langle f\left(\boldsymbol{X}_{t}, t\right), f\left(\boldsymbol{X}_{t}, t\right)\right\rangle$. This provides us a simple scheme for the estimation of the uncertainty directed along the iso-photometric curves' normals. However, in the case of an anisotropic noise model, the uncertainty along the tangent is also needed.

\section{B. Estimation of $\sigma_{\tau}$}

It is not possible to estimate the uncertainty along the photometric contours in a similar way since, as shown in (11), this quantity does not appear in the noise associated to the luminance variation and therefore is not involved in the corresponding quadratic variations. Writing the Itô diffusion associated to the velocity projected along the tangent yields

$$
\boldsymbol{v}_{\text {obs }}^{T} \boldsymbol{\tau}=\boldsymbol{v}\left(\boldsymbol{X}_{t-1}, t-1\right)^{T} \boldsymbol{\tau} d t+\sigma_{\tau}\left(t, \boldsymbol{X}_{t}\right) d B_{t}^{\tau}
$$

This scalar product constitutes a scalar Gaussian random field of mean $\mu=\boldsymbol{v}\left(\boldsymbol{X}_{t-1}, t-1\right)^{T} \boldsymbol{\tau}$ (assuming $\boldsymbol{v}(\boldsymbol{x}, t)$ is a bounded variation process) and covariance $\left(\operatorname{diag}\left(\sigma_{\tau}\right)\right.$ ). We assume that the scalar product $\boldsymbol{v}^{T} \boldsymbol{\tau}$ and the tangent uncertainty $\sigma_{\tau}(t, \boldsymbol{x})$ are sufficiently smooth in space and can be respectively well approximated by the local empirical mean and variance over a local spatial neighborhood $N(\boldsymbol{x})$ of point $\boldsymbol{x}$ :

$$
\begin{aligned}
\mu & =\frac{1}{|N(\boldsymbol{x})|} \sum_{\boldsymbol{x}_{i} \in N(\boldsymbol{x})}\left(\boldsymbol{v}_{\boldsymbol{o b s}}\left(\boldsymbol{x}_{i}, t-1\right)^{T} \boldsymbol{\tau}\right), \\
\sigma_{\tau}^{2} & =\frac{1}{|N(\boldsymbol{x})|-1} \sum_{\boldsymbol{x}_{i} \in N(\boldsymbol{x})}\left(\boldsymbol{v}_{\boldsymbol{o b s}}\left(\boldsymbol{x}_{i}, t-1\right)^{T} \boldsymbol{\tau}-\mu\right)^{2} .
\end{aligned}
$$

The relations (14) and (15) provide new models for the variation of the image luminance under isotropic or anisotropic uncertainties. In this section we have presented a technique to estimate such uncertainties from an available velocity field. The next section focuses on application of those extended brightness consistency models for motion estimation.

\section{APPliCATION OF THE PROPOSED LUMinANCE MODELS}

This section aims at defining a simple local motion estimator that embeds the proposed evolution models as an observation term. As previously indicated the quadratic variation of the bounded variation term of the luminance function is null. We have thus:

$$
\mathbb{E}\left(\mathcal{H}^{2}(f, \boldsymbol{v})\right)=g_{\boldsymbol{\Sigma}} * \mathcal{H}^{2}(f, \boldsymbol{v})=0
$$

As the classical Optical Flow Constraint Equation -OFCE- based on (1), an observation model based on this constraint is ill posed. Similarly to the well-known Lucas-Kanade estimator, we cope this difficulty by assuming constant flow within a Gaussian windowing function defined by the uncertainty estimate. However, for small uncertainty this model will be likely to be still subject to the aperture problem. We introduce hence an additional uncertainty isotropic function of variance $\sigma^{\ell}$, which 


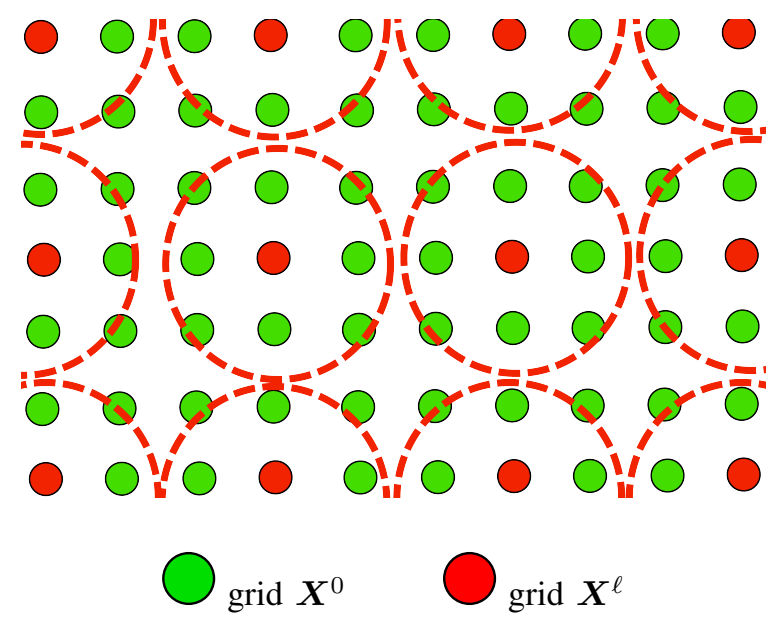

Fig. 2. Multiresolution grids represented through an isotropic Brownian process. Green circles represent the original pixel grid whereas the red ones represent the grid at a given resolution $\ell$.

defines the minimal windowing function at which the estimation is performed. As a result of the locally spatially constant approximation of the motion field, constraint (24) can only be approached, we seek thus to fullfill it in the least square sense. The sought motion estimate $\boldsymbol{v}$ should thus minimize:

$$
\min _{\boldsymbol{v}} g_{\sigma^{\ell}} * g_{\boldsymbol{\Sigma}} *\left(\boldsymbol{\nabla} f \cdot \boldsymbol{v}+\frac{\partial f}{\partial t}+\mathcal{F}(f)\right)^{2},
$$

which yields the following relation, for any position $x$ :

$$
\begin{aligned}
\left(g_{\sigma^{\ell}} * g_{\boldsymbol{\Sigma}} *\left[\begin{array}{cc}
f_{x}^{2} & f_{x} f_{y} \\
f_{x} f_{y} & f_{y}^{2}
\end{array}\right]\right) & \boldsymbol{v} \\
& -g_{\sigma^{\ell}} * g_{\boldsymbol{\Sigma}} *\left(\mathcal{F}(f)+f_{t}\right)\left[\begin{array}{c}
f_{x} \\
f_{y}
\end{array}\right] .
\end{aligned}
$$

Let us note that in our model the Gaussian windowing function can be interpreted as the distribution of a new isotropic constant uncertainty term related to the grid resolution and independent of the motion uncertainties depending on the image data. In practice, the choice of the convolution kernel $\sigma^{\ell}$ is crucial: a large value of $\sigma^{\ell}$ will remove all details whereas a small value is likely to be unstable and may leads in an extreme case to an ill posed problem. In addition, such motion estimation procedure, based on a linearized version of the displaced frame difference, leads to inaccurate measurements of large displacements when the linear assumption of the brightness consistency breaks (high photometric gradients and/or large displacements). To prevent such limitations many authors have proposed to embed such estimation procedures within a pyramidal setup. However, a pyramidal representation requires gaussian filtering, sampling and interpolation of the input data which is likely to introduce artifacts that spoils the estimation. Interpreting the windowing function convolution as associated to the computation of the expectation of a grid uncertainty random term will allows us defining an original continuous multiresolution framework. This is presented in the next paragraph.

1) Multiresolution: A multiresolution scheme consists in redefining the problem on a grid $\boldsymbol{X}^{\ell}$ which can be viewed as a coarse representation of the initial grid $\boldsymbol{X}^{0}=\boldsymbol{X}$ with a Brownian isotropic uncertainty of constant variance $\sigma^{\ell}$. This is illustrated in figure 2: from the initial pixel grid $\boldsymbol{X}^{0}=\boldsymbol{X}$ that corresponds to the plain circles, the problem is redefined on a grid $\boldsymbol{X}^{\ell}$ (represented by the dotted circles) that is a coarse representation of $\boldsymbol{X}$. This reads:

$$
\boldsymbol{X}^{\ell}=\boldsymbol{X}^{0}+\sigma^{\ell} \mathbb{I}_{2} d \boldsymbol{B} .
$$

The motion $\boldsymbol{v}\left(\boldsymbol{X}_{t-1}^{\ell}, t-1\right)$ on this grid should minimize the expectation $E\left(\mathcal{H}^{2}(f, \boldsymbol{v}) \mid \boldsymbol{X}^{0}\right)$ which is equivalent (see appendix A) to a convolution of $\mathcal{H}^{2}(f, \boldsymbol{v})$ with the isotropic gaussian $\mathcal{N}\left(O, \sigma^{\ell}\right)$. Therefore, one gets exactly the system in (25) which is solved locally by inverting the system in (26). A main advantage of such a formulation of the multiresolution setup is to naturally get rid of the use of a pyramidal image representation. Instead of dealing with successive decimations of factor 2 of the initial image to fix the different multiresolution levels, the evolutions of the levels $\ell$ are much flexible here. This framework may be interpreted as a scale space representation of the extended brightness consistency assumption [20]. However in our case it stems rigorously from an uncertainty analysis of the point location.

With all these elements, we can define the incremental local motion estimation technique presented in the next paragraph. 
2) Incremental framework: The local estimator proposed here comprises a specific multiresolution scheme where at each level, an incremental framework is defined to estimate as accurately as possible the uncertainties. The following algorithm is used:

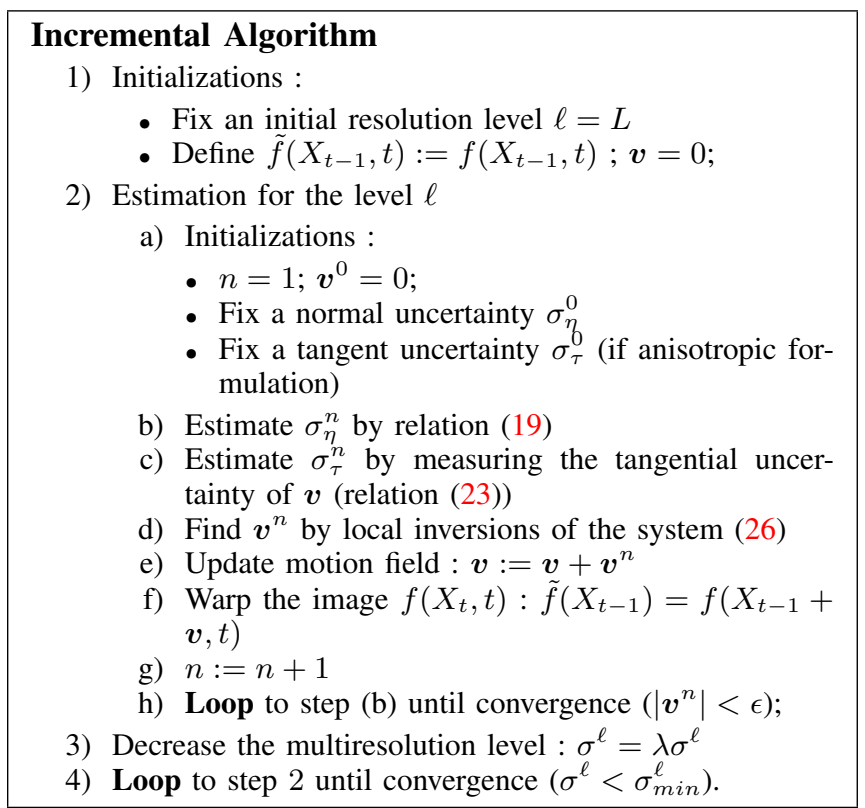

The previous framework is a natural and simple implementation of a local motion estimation technique using the proposed models for the evolution of the luminance. A quantitative and qualitative evaluation of such an estimator, with comparisons to the classic OFCE will be presented in section V.

\section{EXPERIMENTAL RESULTS}

We present in this section some experimental results of the local motion estimator described in section IV. We show examples on synthetic fluid images and on the Middleburry database ${ }^{1}$. It is important to outline that the estimator defined in section IV constitutes a local technique whose aim is simply to valid, compare and qualify the observation model based on stochastic uncertainties $v s$ the usual OFCE (1). Hence, its performances have to be compared to other local approaches.

Following the proposed algorithm, four uncertainty parameters have to be set: $\sigma_{\eta}^{0}$ and $\sigma_{\tau}^{0}$ (initial value of the uncertainties), $\sigma^{\ell}$ (coarse scale level) and $\sigma_{\text {min }}^{\ell}$ (finest scale level). In practice we have fixed $\sigma_{\eta}^{1}=\sigma_{\tau}=1, \sigma^{\ell}=40$ and $\sigma_{\min }^{\ell}=7$. As for the multiscale settup, the parameter $\lambda$ that rules the scale decrease $\left(\sigma^{\ell+1}=\lambda \sigma^{\ell}, \sigma^{\ell+1}>\sigma_{\text {min }}^{\ell}\right)$ has been set to $\lambda=0.3$. We can note that a standard pyramidal scheme would correspond to $\lambda=0.5$. In practice we have founded, as in [37], that smaller values were more efficient. This is illustrated in figure 4. As a first benchmark we analyze the results obtained on images depicting the evolution of a $2 \mathrm{D}$ turbulent fluid flow.

\section{A. Fluid images}

In fluid imagery, it is common to visualize and analyze flows with particles: the fluid flow is seeded with small particles and enlighten through a laser sheet. All existing commercial systems are local motion estimators based on correlation techniques. These technique are usually referred as PIV (Particles image velocimetry) methods.

We used a pair of synthetic images of $256 \times 256$ pixels obtained by DNS (Direct Numerical Simulation of Navier-Stokes equations) and representing a 2D turbulent flow. Numerical values of average angular error (AAE) [4] and of the Root Mean Square Error (RMSE) are used as criteria to compare our estimators (isotropic and anisotropic) with some of the state-of-the-art approaches. These results are depicted in table I. The techniques to which the proposed estimators are compared are :

- an Horn \& Schunck estimator (HS) [16]

- a commercial software based on correlation (DaVis 7.2 from LaVision GmbH, technique COM)

- a pyramidal incremental implementation of the Lucas-Kanade estimator (LK) [21]

- the proposed framework in section IV with the OFCE as an observation model (OFCE) (i.e with a zero uncertainty)

- two fluid-dedicated dense motion estimators based on a Div-Curl smoothing with different minimization strategies (DC1DC2, [11], [39]).

- a fluid-dedicated dense motion estimator based on a turbulence subgrid model in the data-term (TUR, [9]).

${ }^{1}$ http://vision.middlebury.edu/flow/ 


\begin{tabular}{|c|ccccccccc|}
\hline & LK & COM & HS & DC 1 & DC 2 & TUR & OFCE (57s) & ISO (63s) & ANISO (81s) \\
\hline AAE & $6.07^{\circ}$ & $4.58^{\circ}$ & $4.27^{\circ}$ & $4.35^{\circ}$ & $3.04^{\circ}$ & $4.49^{\circ}$ & $4.53^{\circ}$ & $3.59^{\circ}$ & $3.12^{\circ}$ \\
RMSE & 0.1699 & 0.1520 & 0.1385 & 0.1340 & 0.09602 & 0.1490 & 0.1243 & 0.1072 & 0.0961 \\
\hline
\end{tabular}

TABLE I

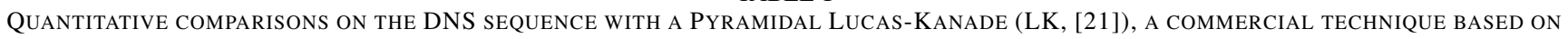
CORRELATION (COM, LA VISION SYSTEM), HORN \& SCHUNCK (HS, [16]), TWO FLUID DEDICATED MOTION ESTIMATORS WITH DIV-CURL SMOOTHING TERMS (DC 1 : [11]; DC2 : [39]), A FLUID DEDICATED MOTION ESTIMATOR WITH TURBULENCE SUB-GRID MODELS IN THE DATA TERM (TUR, [9]), OUR APPROACH USING THE CLASSIC OPTICAL-FLOW CONSTRAINT EQUATION (OFCE), OUR APPROACH IN ISOTROPIC (ISO) AND ANISOTROPIC (ANISO).

The pyramidal Lucas and Kanade implementation differs only from the multiscale estimator with zero uncertainty (OFCE) in the setting of the decreasing scale parameter to $\lambda=0.5$. For techniques issued from our local framework (OFCE, ISO and ANISO), we have added the run time experiments. The estimation was performed using matlab on a 4Go RAM PC of $2.6 \mathrm{GHz}$. As for running times, it turns out, as expected, that the anisotropic version is longer. This is due to the local convolutions (with various standard deviations) required by the technique that can not be as efficiently implemented as an isotropic convolution with constant standard deviation. However it is very important to outline that this technique is local and therefore, at each iteration, all local estimations (step 2-d of the algorithm) can be parallelized.

In figure 3, we present an image of the sequence, the estimated flow with the proposed method (anisotropic version) and the error flow field. We have also plotted in figure 5 the velocity spectra of the different techniques and compared them with the ground truth. These spectra are represented in log-log coordinates (figure 5(a)) and a standard-log coordinate system (figure $5(\mathrm{~b}))$ in order to highlight the accuracy on small and large scales respectively.

On table I, one can immediately observe that compared to the other local approaches, our method provides very good results since the global accuracy is highly superior than the Lucas-Kanade (LK) and the commercial software (COM). Compared to dense techniques (HS, DC1 and DC2), our numerical results are in the same order of magnitude which is a very relevant point. They are competitive with some dense estimation techniques dedicated to fluid flows analysis (DC1-DC2, [11], [39]). The comparison between the results OFCE, ISO and ANI is very interesting since it highlights the benefit of the stochastic formulation of the image luminance. These three results have been estimated with the same incremental estimation framework of section IV but using an observation model based respectively on the usual OFCE (equation (1), technique OFCE), an isotropic uncertainty (equation (14), technique ISO) and an anisotropic uncertainty (equation (15), technique ANISO). From the corresponding quantitative errors it is obvious that the uncertainty modeling greatly improves the results, especially in the anisotropic approach, with however a higher computational cost.

In order to evaluate the influence of the decreasing parameter $\lambda$ in the multiresolution process (step 3 of the incremental algorithm), we have plotted in figure 4 the angular error obtained on this pair of images with the ANI algorithm depending on several values of $\lambda \in[0.1,0.9]$. As one can observe, the minimum is reached for $\lambda \approx 0.3$ and not around $\lambda=0.5$ which corresponds to the usual pyramidal framework. The same observations hold for other kind of images.

If now one observes the spectra of the velocity shown in figure 5, we see that the small scales (right part of the graph) are much better recovered by the proposed estimators than by the dense estimators. They are generally difficult to estimate and often smoothed out with the spatial regularizers introduced in the dense techniques. Even if the Lucas-Kanade technique seems to exhibit better results on small scales, when observing the figure 5(b), it is obvious to note that large scales are badly estimated with this approach and this yields a very poor overall accuracy (see table I). As for the large scales the results are comparable with the best dense dedicated techniques. We believe hence that our estimator constitutes an appealing alternative to usual local PIV methods. Let us now describe the accuracy of the observation term on some images of the Middleburry database.

\section{B. Middleburry database}

The Middleburry database has recently been proposed in [2] to compare recent and state-of-the-art optical-flow methods. It contains several sequences with various challenging situations like hidden textures, complex scenes, non rigid motion, high motion discontinuities, ... The aim of this validation is

1) to highlight the benefit of the proposed brightness consistency model under location uncertainty. To that end, we compare the results obtained with the simple motion estimator presented in the previous section using three observation terms : the proposed ones in their isotropic and anisotropic versions and the usual OFCE (i.e. without uncertainty);

2) to promote the interest of the associated maps of uncertainties by demonstrating that they are directly related to the quality of the estimated motion field. To that end, we analyze the evolution of the error on the motion field when this latter is computed on points corresponding to low values of uncertainties.

On this database, we have first detailed the algorithm on pair of images where a ground truth is available (since we need to compare the results on sparse motion fields). In order to help the reader in understanding the approach, in a second step, we have applied the technique on the whole database (even if the aim if to propose an alternative observation model only). 


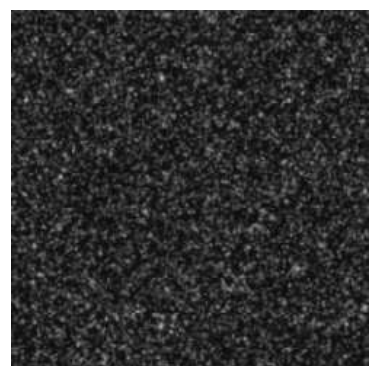

(a)

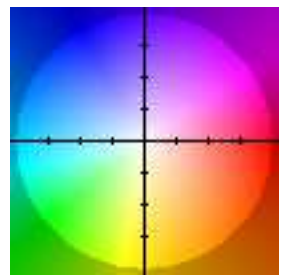

(e)

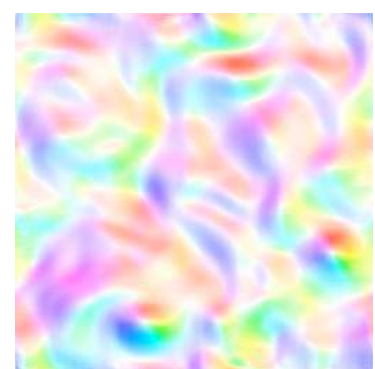

(b)

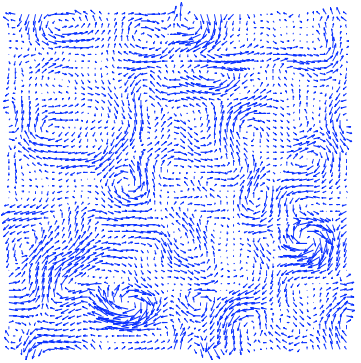

(f)

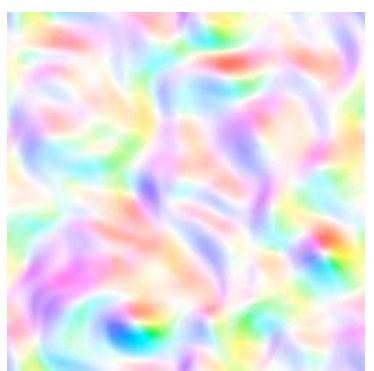

(c)

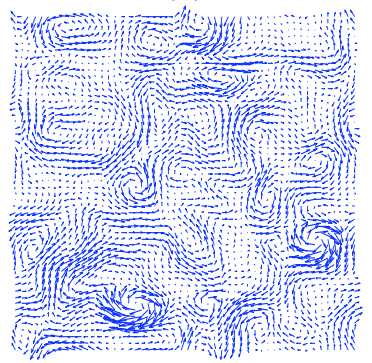

(g)

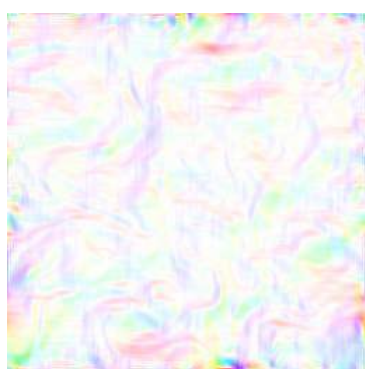

(d)

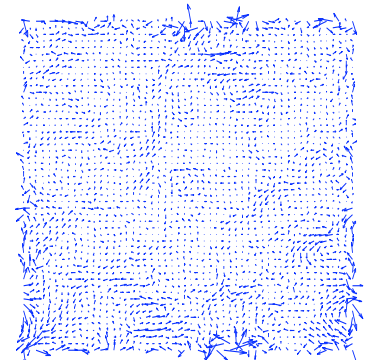

(h)

Fig. 3. Results on the DNS sequence : Top (a): an image of the sequence; (b): the estimated flow ; (c): the real flow; (d): the difference flow represented with the coding color in (i); Bottom (e): the coding color vor vector flow representation; (f-g-h) vector representation of the estimated motion field, the ground truth and the difference field $(\times 25)$.

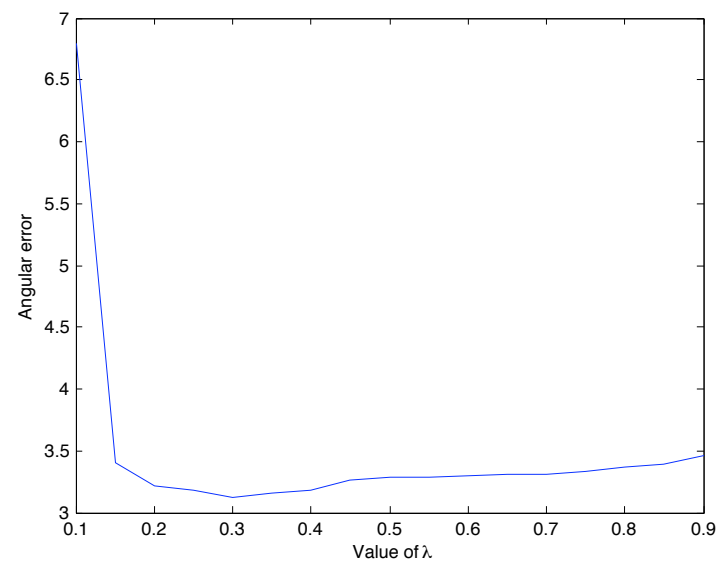

Fig. 4. Influence of the decreasing parameter $\lambda$ in the multiresolution process. Results of the angular error obtained for different values of $\lambda$ using the ANI algorithm on the DNS sequence.

\section{Evaluation of data with ground truth}

We have tested our approaches on the "Dimetrodon" and "Venus" sequences. For these sequences a ground truth and a comparison with others state-of-the-art approaches are available. One image of each sequence is depicted in figures 6 and 8 (a) with the associated ground truth in figures 6 and 8 (b). The first sequence exhibits large homogeneous motions with very located discontinuities (around the dinosaur) whereas the discontinuities of the Venus sequence are more spatially homogeneous.

The quantitative results and associated run times are presented in the table II. When comparing the three first columns that use exactly the same technique but based on the usual OFCE (relation (1)), our luminance model with isotropic (ISO, relation (14)) and anisotropic (ANISO, relation (15)) uncertainties, it immediately points out that the proposed models enable to enhance significantly the quality of the results. This fair comparison of the three observation models onto the same estimator promotes the use of a stochastic formulation under anisotropic uncertainties. In fact this latter version is a softer constraint than the OFCE which, as shown previously, assumes implicitly a perfect measurement without any incertitudes. This better efficiency results in a larger time computation. However as already mentioned, such local estimator can efficiently be implemented using parallel softwares.

The estimated motion fields under the anisotropic luminance formulation are represented on figures fig.6(d) and fig.8 (d) and can be compared with the ground truth on fig.6(c) and fig.8(c). 


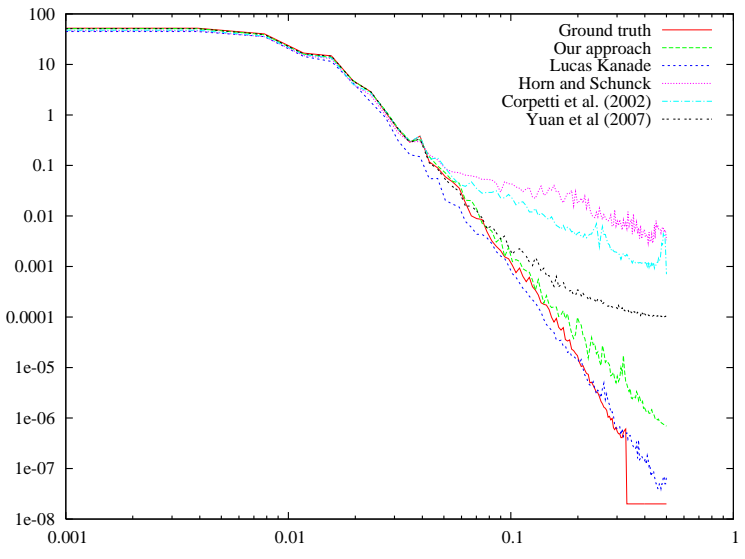

(a)

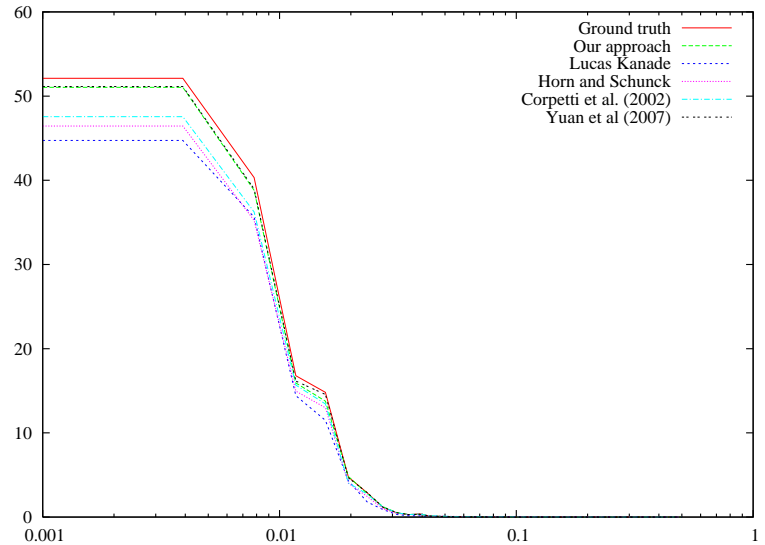

(b)

Fig. 5. Spectra of the velocity compared with ground truth and for several method (a): log-log representation (highlights small scales on the right part) and (b): non log-log representation (highlights large scales on the left part). Color are : Red : ground truth; Green : our approach (anisotropic version); Blue : Lucas-Kanade [21]; Purple : Horn and Schunck [16]; Cyan: Div-Curl smoothing [11] and Black: Div-Curl in mimetic discretization [39] .

The benefit of this new formulation of the luminance (ANISO version) compared to the usual brightness consistency assumption (OFCE version) is demonstrated quantitatively in table II. In order to visually interpret these improvements, we have depicted in figures fig.6(e-f) and fig.8(e-f) the difference motion fields between the OFCE estimator and the ground truth (in fig.6-8(e)) and the difference between ANISO and OFCE estimators (in fig.6-8(f)). By comparing figures 6-8(c) and figures $6-8(\mathrm{e})$, one can observe that the errors diffuse on larger areas using the OFCE than with our anisotropic version of the OFCE. This is more remarkable in figure $8(\mathrm{e})$ where it is obvious that the error areas in red (bottom left part of the image), blue (bottom-middle) and yellow (middle) are much larger with the OFCE. The differences ANISO-OFCE in fig.6-8(f) highlight the areas where the proposed estimator differs significantly from the OFCE. As expected, the benefit appears in areas where the luminance exhibits high discontinuities whereas homogeneous areas generate more or less the same behavior of both estimators.

Let us in addition remind that the motion estimation technique that has been developed for comparing the models of luminance is quite simple (based on the Lucas and Kanade estimator). Therefore, as expected, the errors are mainly localized on discontinuities. Since the Venus sequence contains more discontinuity areas than the Dimetrodon one, our results are less performing on this pair of images. However it is very informative to observe that despite the simplicity of this technique, our results in table II are very competitive and sometimes outperform advanced dense techniques with a specific process for motion discontinuities recovery. Apart from regions exhibiting motion discontinuities and where the error can be important, the difference fields of fig.6(c) and fig.8(c) reveal very good results (white areas) in the other locations. This suggests that the luminance models introduced in this paper is useful in allowing a global improvement of accuracy.

Additionally to the estimated motion fields, such a technique is able to extract the associated uncertainty areas. For each location of the image, these areas represent the local neighboring where the brightness consistency assumption is likely to hold and on which the motion is locally estimated. We have plotted in fig. 7(a) and fig.9(a), for a given location of the image, such anisotropic areas, in practice computed with relations (19) and (23). As one can observe, they correspond to the main structures of the image (mainly anisotropic along the contour and more isotropic in homogeneous regions) and thus provide to the user valuable information. Therefore, in a step forward, these areas can be taken into account in a more advanced motion estimation technique in order to better preserve the discontinuities.

The norm of the global uncertainty $\sqrt{\sigma_{\eta}^{2}+\sigma_{\tau}^{2}}$ maps obtained at the end of the process with the best estimator (the anisotropic one) are plotted in fig.7(b) and fig.9(b). As expected, homogeneous areas where the aperture problem holds correspond to high values of $\sigma_{\eta}$ whereas small values are linked to photometric contours. Such output of our method is very promising since it it highlights the main structures of the images and gives an indicator of the quality of the estimation. To justify this last point, we have depicted in fig.7(c) and fig.9(c) the reconstructed errors when we take into account for the evaluation only the points where the incertitude is bellow a given value (blue lines) and the corresponding percentage of points used for the computation (red lines). In the two cases, the error grows with the uncertainty, which indicates that it is a reliable indicator of the quality of the measurements.

We then strongly believe that the stochastic models presented can be exploited in the future to design dense estimators relying on the proposed brightness consistency model.

Let us now turn to some experiments on the complete Middleburry database.

Evaluation on the complete database

In a second step, we have applied the proposed local estimator in its anisotropic version (since it performs better on images with 
TABLE II

QUANTITATIVE RESUlts, TIME COMPUTATION AND COMPARISONS on the Dimetrodon, Yosemite AND VenUS SEQUENCE

Results on the Dimetrodon sequence $(584 \times 388$ pixels $)$

\begin{tabular}{|c|c|c|c|c|c|c|c|c|}
\hline Method & OFCE (124s) & ISO (208s) & ANISO (271s) & Bruhn et al. & Black, Anandan & Pyramid LK & Media Player $^{\mathrm{TM}}$ & Zitnick et al \\
\hline Ang. error & $7.95^{\circ}$ & $3.95^{\circ}$ & $2.85^{\circ}$ & $10.99^{\circ}$ & $9.26^{\circ}$ & $10.27^{\circ}$ & $15.82^{\circ}$ & $30.10^{\circ}$ \\
\hline \multicolumn{9}{|c|}{ Results on the Venus sequence $(420 \times 380$ pixels $)$} \\
\hline Method & OFCE (93s) & ISO (156s) & ANISO (197s) & Bruhn et al. & Black, Anandan & Pyramid LK & Media Player $^{\mathrm{TM}}$ & Zitnick et al \\
\hline Ang. error & $12.02^{\circ}$ & $10.23^{\circ}$ & $8.42^{\circ}$ & $8.73^{\circ}$ & $7.64^{\circ}$ & $14.61^{\circ}$ & $15.48^{\circ}$ & $11.42^{\circ}$ \\
\hline
\end{tabular}

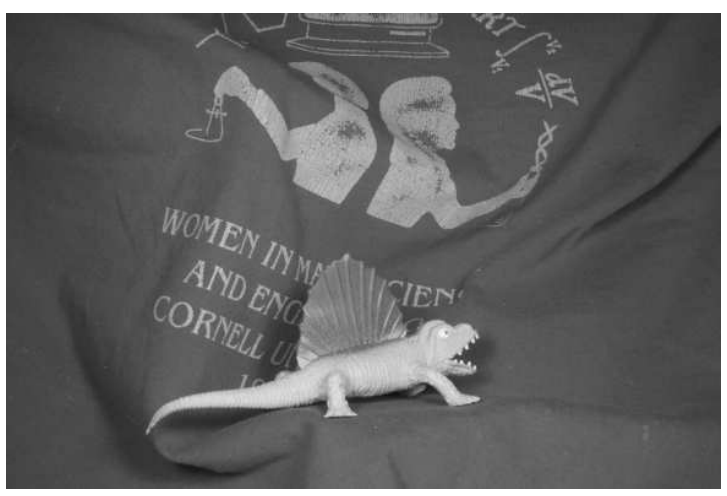

(a): the first image

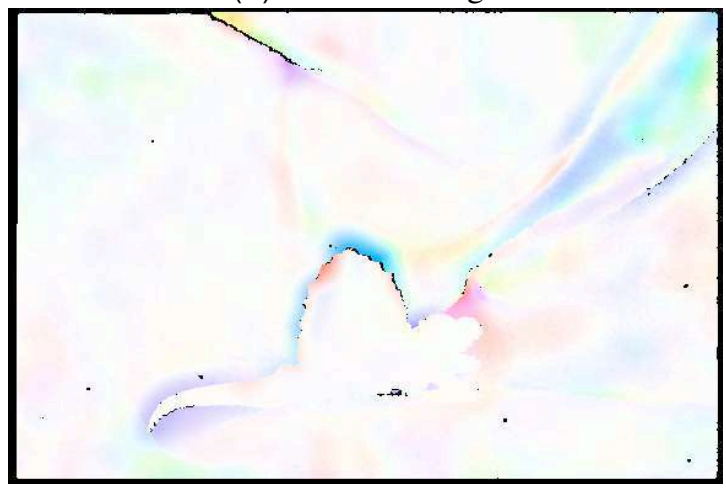

(c):difference (ANISO - ground truth)

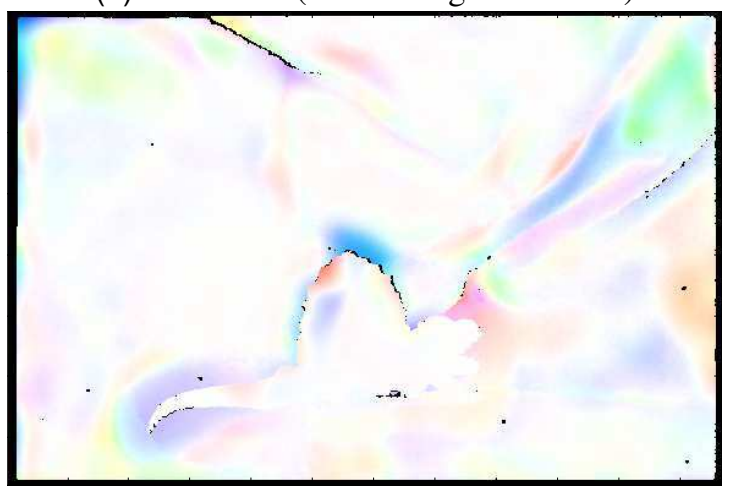

(e): difference (OFCE - ground truth)

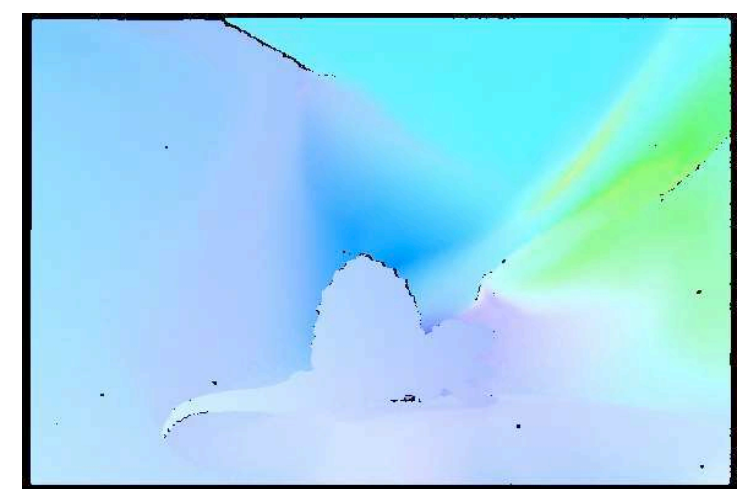

(b): ground truth

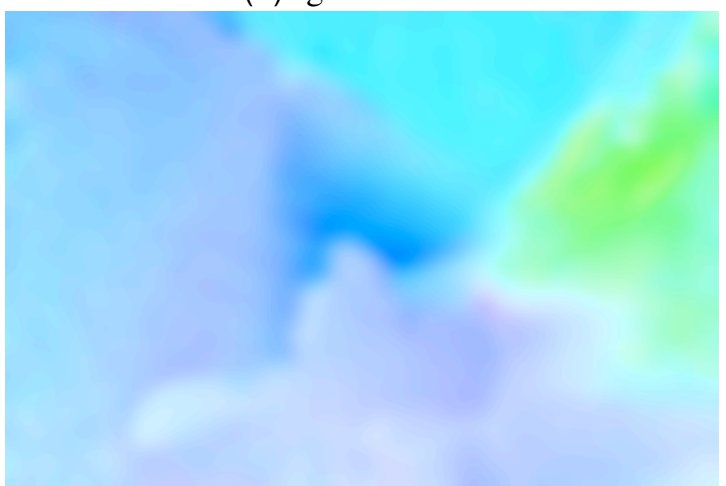

(d): our motion field

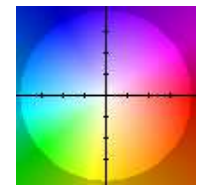

color coding

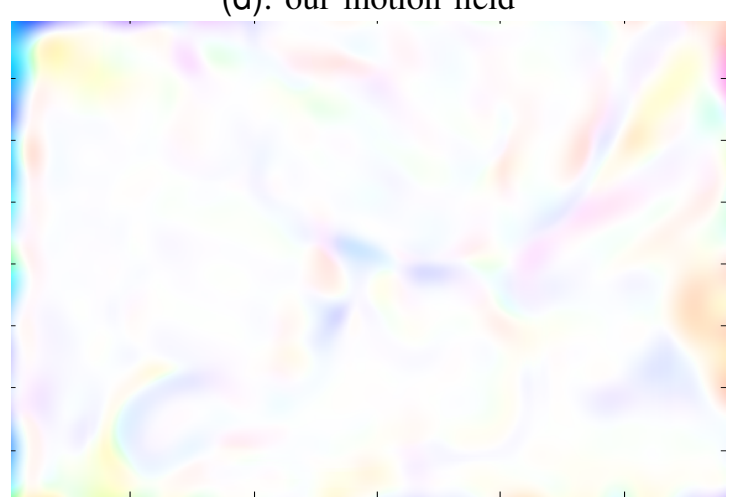

(f): difference (ANISO-OFCE)

Fig. 6. Dimetrodon sequence (a): an image of the sequence; $(\mathrm{b})$ : the ground truth; $(\mathrm{c})$ : difference estimated velocity field and ground truth; (d): the estimated motion field with our approach in anisotropic version (ANISO); (e-f): differences (OFCE - ground truth) and (ANISO-OFCE) 


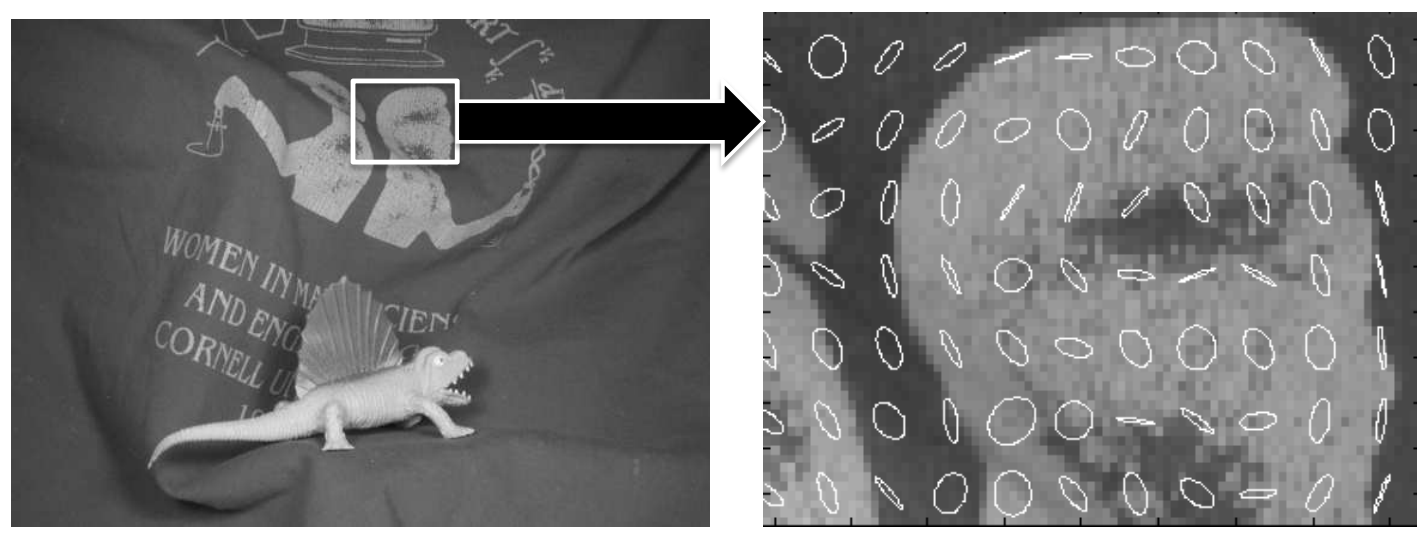

(a): representation of some uncertainty areas

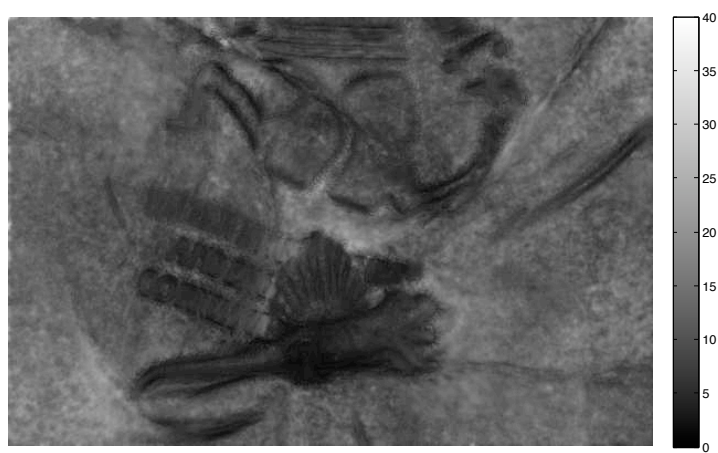

(b); uncertainty map
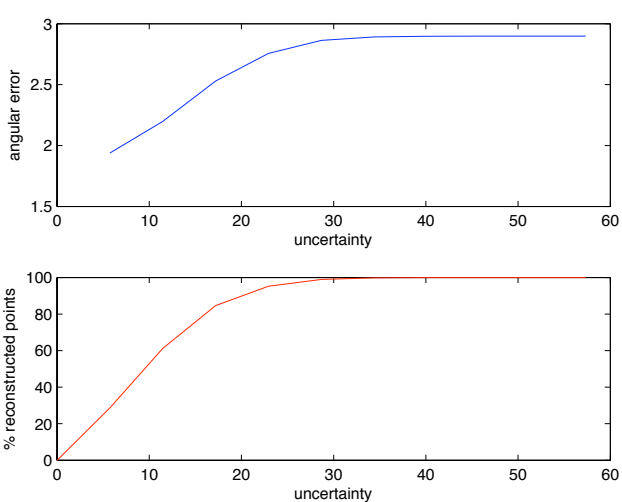

(c)

Fig. 7. Uncertainty areas for the Dimetrodon sequence: (a): a snapshot of some anisotropic uncertainty areas highlighted by the ANISO technique on which one can observe that the optical flow is computed depending on the photometric level-lines of the luminance; (b): the extracted uncertainty map $\sigma_{\eta}$ and (c): evolution of the error and percentage of correct motion fields when one takes into account only velocity fields with smaller values of $\sqrt{\sigma_{\eta}^{2}+\sigma_{\tau}^{2}}$

ground truth) on the evaluation dataset. A snapshot of numerical results is depicted in figure 10. Our method is named "SLK" for Stochastic Lucas-Kanade. One can observe that the presented technique is the best in the category of "local estimators" since it outperforms the Pyramid Lucas-Kanade and the FOLKI estimator, this latter being based on a local window registration [18]. In addition, it is promising to observe that it is more efficient that some dense techniques, as the one named "Adaptive flow" with adaptive smoothness priors.

Of course, its efficiency is poorer than sophisticated optical-flow approaches equipped with advanced smoothing terms since only a coarse estimation corresponding to the uncertainty level $\sigma_{\min }^{\ell}$ is available (without any smoothing). Therefore, all discontinuities are completely smoothed out. Nevertheless, these experiments prove that the introduction of uncertainty models in the data term enhances the quality of the estimation since comparable local estimators based on the OFCE are less competitive. To enforce the relevance of the observation model, we should note that on sequences where the flow possesses softer discontinuities like Yosemite or Dimetrodon, the quality grows and outperforms several dense methods. All results can be seen in the Middleburry web-site ${ }^{2}$.

\section{CONCLUSION}

In this paper an observation model for optical flow estimation has been introduced. The new operator is based on a stochastic modeling of the brightness consistency uncertainty. This data model constitutes a natural extension of the usual brightness consistency assumption. Isotropic and anisotropic uncertainty models have been presented. From this new data term, we have designed a simple local motion estimator where the multiresolution is also interpreted in term of a spatial uncertainty.

The performances of this local estimator have been validated on synthetic fluid flows issued from Direct Numerical Simulations and on the Middleburry synthetic database. In the first case, the results have exhibited significant performances, especially in the recovery of small scales that are generally smoothed out by spatial regularizers of dense approaches. As for the Middleburry database, the simple local implementation of the presented data-term outperforms local approaches. We

\footnotetext{
${ }^{2}$ http://vision.middlebury.edu/flow/eval/results/results-e1.php
} 


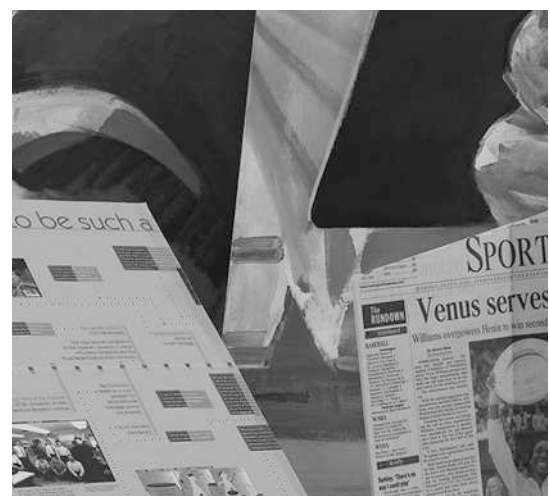

(a): the first image

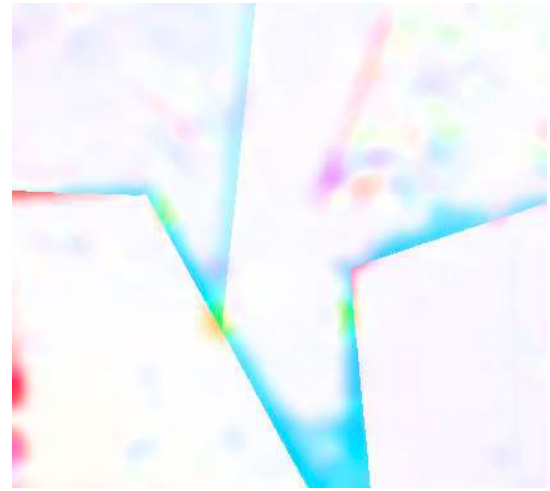

(c):difference (ANISO - ground truth)

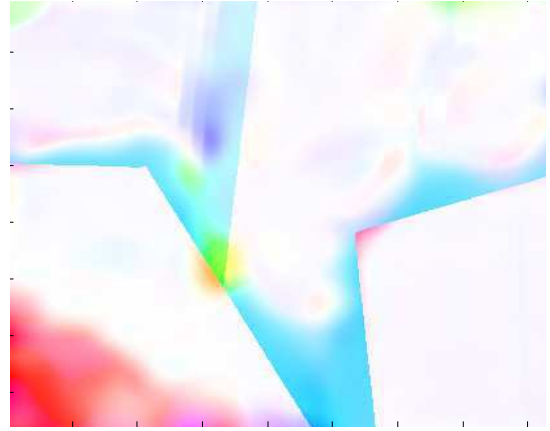

(e): difference (OFCE - ground truth)

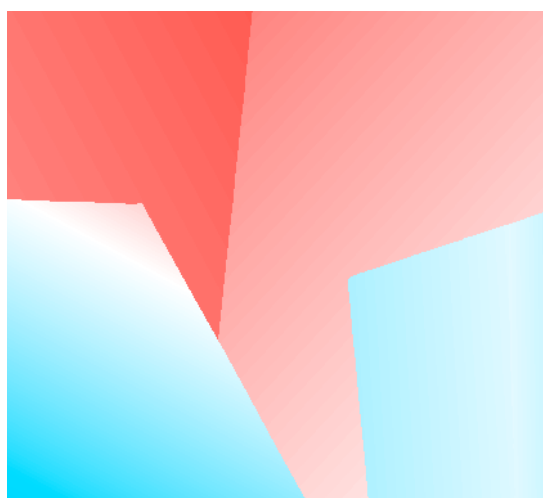

(b): ground truth

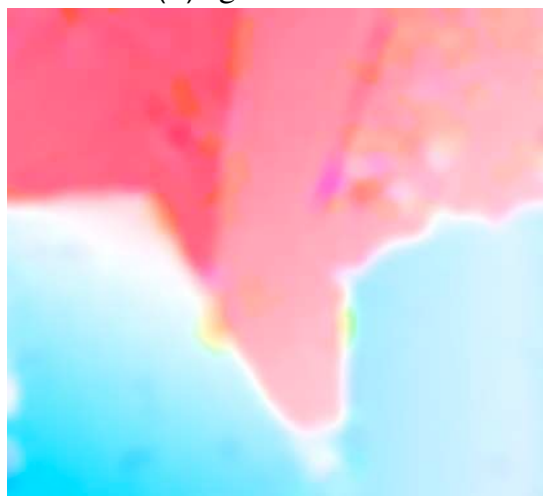

(d): our motion field

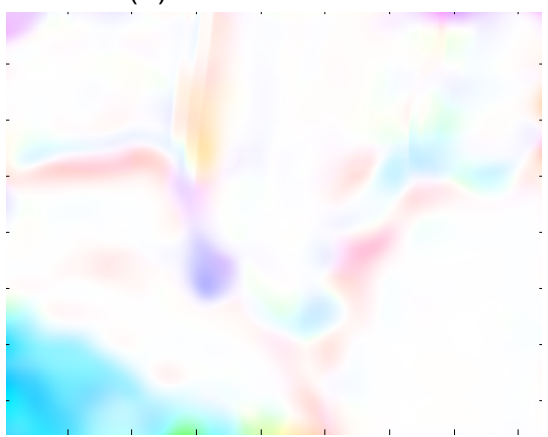

(f): difference (ANISO-OFCE)

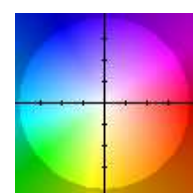

color coding

Fig. 8. Venus sequence (a): an image of the sequence; (b): the ground truth; (c): difference estimated velocity field and ground truth; (d): the estimated motion field with our approach in anisotropic version (ANISO); (e-f): differences (OFCE - ground truth) and (ANISO-OFCE)

therefore believe that this stochastic modeling is a very promising alternative to the usual deterministic OFCE for all optical-flow methods.

\section{APPENDIX}

The conditional expectation given $\boldsymbol{X}_{t-1}$ of any function $\Psi\left(\boldsymbol{X}_{t}, t\right)$ of a stochastic process defined through Itô diffusion (3) and discretized through an Euler scheme $\boldsymbol{X}_{t}=\boldsymbol{X}_{t-1}+\boldsymbol{v}\left(\boldsymbol{X}_{t-1}\right) d t+\boldsymbol{\Sigma}^{1 / 2}\left(B_{t+1}-B_{t}\right)$ may be written as:

$$
E\left(\Psi\left(\boldsymbol{X}_{t}, t\right) \mid \boldsymbol{X}_{t-1}\right)=\int_{\mathbb{R}} \Psi\left(\boldsymbol{X}_{t}, t\right) p\left(\boldsymbol{X}_{t} \mid \boldsymbol{X}_{t-1}\right) d \boldsymbol{X}_{t}
$$

As the process $\boldsymbol{X}_{t}$ is known up to the Brownian motion $\boldsymbol{\Sigma} d \boldsymbol{B}_{t}$, the probability $p\left(\boldsymbol{X}_{t} \mid \boldsymbol{X}_{t-1}\right)$ is a multidimensional Gaussian of variance $\boldsymbol{\Sigma} \sqrt{d t}(d t=1$ here) and we get:

$$
\begin{aligned}
& E\left(\Psi\left(\boldsymbol{X}_{t}\right) \mid \boldsymbol{X}_{t-1}\right)=\frac{1}{\sqrt{2 \pi} \operatorname{det}(\boldsymbol{\Sigma})^{\frac{1}{2}}} \int_{\mathbb{R}} \Psi\left(\boldsymbol{X}_{t}, t\right) \\
& \exp \left(-\left(\boldsymbol{X}_{t-1}+\boldsymbol{v}-\boldsymbol{X}_{t}\right) \boldsymbol{\Sigma}^{-1}\left(\boldsymbol{X}_{t-1}+\boldsymbol{v}-\boldsymbol{X}_{t}\right)\right) d \boldsymbol{X}_{t} .
\end{aligned}
$$




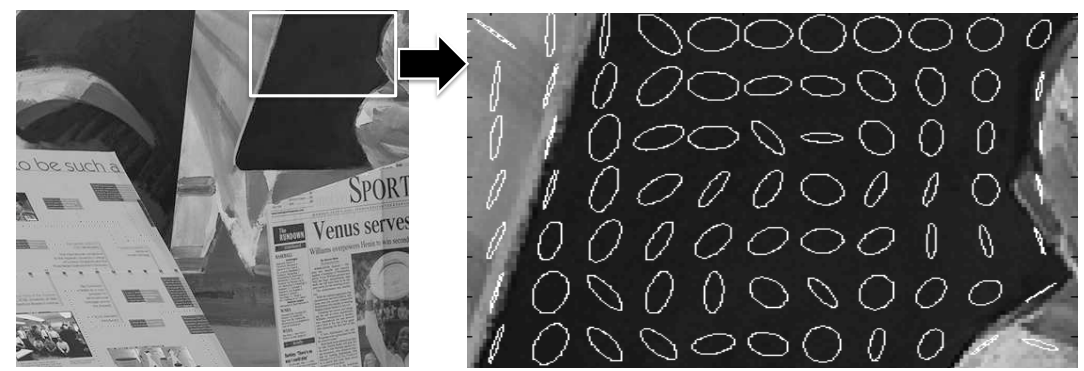

(a): representation of some uncertainty areas

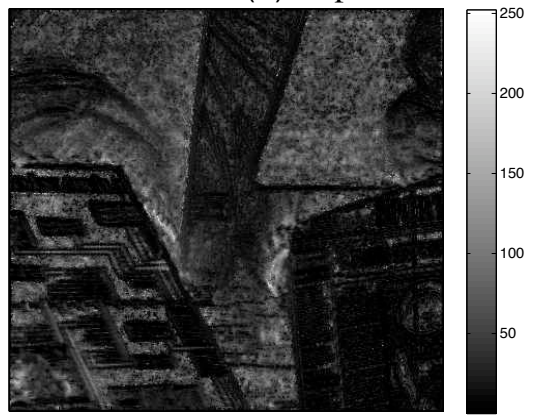

(b); uncertainty map
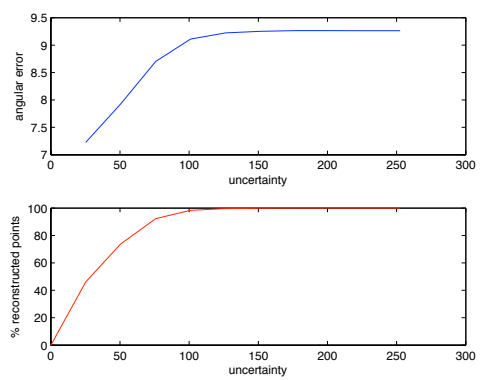

(c)

Fig. 9. Uncertainty areas for the Venus sequence: (a): a snapshot of some anisotropic uncertainty areas highlighted by the ANISO technique on which one can observe that the optical flow is computed depending on the photometric level-lines of the luminance; (b): the extracted uncertainty map $\sigma_{\eta}$ and (c): evolution of the error and percentage of correct motion fields when one takes into account only velocity fields with smaller values of $\sqrt{\sigma_{\eta}^{2}+\sigma_{\tau}^{2}}$

\begin{tabular}{|c|c|c|c|c|c|c|c|c|c|}
\hline $\begin{array}{l}\text { Average } \\
\text { angle } \\
\text { error }\end{array}$ & $\begin{array}{l}\text { vg. } \\
\text { ank }\end{array}$ & $\begin{array}{l}\text { Army } \\
\text { (Hidden texture) } \\
\text { GT } \frac{\text { imo }}{\text { all }} \underline{\underline{\text { disc }}} \frac{\underline{\text { untext }}}{}\end{array}$ & $\begin{array}{l}\text { Mequon } \\
\text { (Hidden texture) } \\
\text { GT im0 im1 } \\
\text { all } \underline{\text { disc }} \text { untext }\end{array}$ & $\begin{array}{l}\text { Schefflera } \\
\text { (Hidden texture) } \\
\text { GT } \frac{\text { im0 }}{\text { all }} \underline{\underline{\text { disc }}}\end{array}$ & $\begin{array}{l}\text { Wooden } \\
\text { (Hidden texture) } \\
\text { GT } \\
\text { all } \frac{\text { imo }}{\text { disc }} \frac{\text { im1 }}{\text { untext }}\end{array}$ & 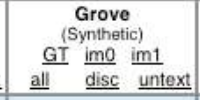 & 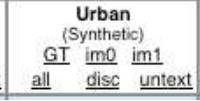 & 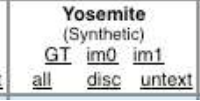 & 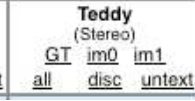 \\
\hline$\pi[0]$ & 3.0 & 64726.05014 .648 . & .34625 .04917 .547 & 84730.14818 .147 & 51 & 49 & 4501 & 31 & \\
\hline Japtive fle & 46.8 & $13.24820 .845 \quad 14.047$ & 17.14822 .04417 .948 & 18.14827 .14422 .851 & 11.84331 .14510 .543 & $\underline{6.35} 507.13506 .2548$ & 9.874821 .8449 .4447 & 12.65111 .45120 .051 & 7.754413 .6417 .7343 \\
\hline LLF & 8.5 & 10.54625 .64911 .946 & 20.95026 .25026 .150 & 17.64531 .15016 .544 & 15.44732 .64716 .047 & $\underline{6.16} 496.53499 .0750$ & $12.25029 .751 \quad 13.050$ & 4.67475 .83479 .4149 & 18.25022 .85025 .15 \\
\hline yramid LK [2] & 49.9 & 13.95020 .94621 .451 & 24.15123 .14530 .251 & 20.95129 .54721 .950 & 22.25034 .65025 .050 & 18.75123 .15120 .251 & 21.25124 .54921 .051 & 6.41507 .024910 .850 & 25.65131 .55134 .55 \\
\hline
\end{tabular}

Fig. 10. Results on the middleburry database quantitative values of the evaluation dataset. One can observe that our method (SLK) is better than other local estimators (Pyramid LK and FOLKI).

By a change of variable $\mathbf{Y}_{t}=\boldsymbol{X}_{t-1}+\boldsymbol{v}-\boldsymbol{X}_{t}$, this expectation can be written as:

$$
\begin{aligned}
& E\left(\Psi\left(\boldsymbol{X}_{t}, t\right) \mid \boldsymbol{X}_{t-1}\right)= \\
& \frac{1}{\sqrt{2 \pi} \operatorname{det}(\boldsymbol{\Sigma})^{1 / 2}} \int_{\mathbb{R}} \Psi\left(\boldsymbol{X}_{t-1}+\boldsymbol{v}-\mathbf{Y}_{t}, t\right) \exp \left(-\mathbf{Y}_{t} \boldsymbol{\Sigma}^{-1} \mathbf{Y}_{t}\right) d \mathbf{Y}_{t} \\
& =\Psi\left(\boldsymbol{X}_{t-1}+\boldsymbol{v}, t\right) * \mathcal{N}(0, \boldsymbol{\Sigma}) .
\end{aligned}
$$

\section{REFERENCES}

[1] E. Arnaud, E. Mmin, and B. Cernuschi-Frias. Conditional filters for image sequence based tracking - application to point tracking. IEEE Trans. Image Processing, 14(1):63-79, 2005.

[2] S. Baker, D. Scharstein, J. Lewis, S. Roth, M. Black, and R. Szeliski. A database and evaluation methodology for optical flow. In Int. Conf. on Comp. Vis., ICCV 2007, 2007.

[3] J. Barron, D. Fleet, and S. Beauchemin. Performance of optical flow techniques. Int. J. Comput. Vis., 12(1):43-77, 1994.

[4] J. L. Barron, D. J. Fleet, S. S. Beauchemin, and T. A. Burkitt. Performance of optical flow techniques. International Journal of Computer Vision, 12(1):43-77, 1994.

[5] F. Becker, B. Wieneke, S. Petra, A. Schröder, and C. Schnörr. Variational adaptive correlation method for particle image velocimetry. IEEE Trans. on Image Processing, 2011. To appear.

[6] M. Black and P. Anandan. Robust incremental optical flow. In J. Eklundh, editor, Proc. Conf. Comp. Vision Pattern Rec., volume 800 of LNCS, pages 296-302. Springer-Verlag, 1992.

[7] T. Brox, A. Bruhn, N. Papenberg, and J. Weickert. High accuracy optical flow estimation based on a theory for warping. In European Conference on Comput. Vis. (ECCV'04). Springer-Verlag, 2004.

[8] A. Bruhn, J. Weickert, T. Kohlberger, and C. Schnoerr. A multigrid platform for real-time motion computation with discontinuity-preserving variational methods. Int. J. Com. Vis., 70(3):257-277, 2006.

[9] C. Cassisa, S. Simoens, and V. Prinet. Two-frame optical flow formulation in an unwarping multiresolution scheme. In Springer-LNCS 5856. Proceedings of the 14th Iberoamerican Conference on Pattern Recognition (CIARP'09), pages 90-797, Guadalajara, Mexico, 2009.

[10] T. Corpetti, E. Mémin, and P. Pérez. Estimating fluid optical flow. In Proc. Int. Conf. Pattern Recognition, volume 3, pages 1045-1049, Barcelona, Spain, September 2000 . 
[11] T. Corpetti, E. Mémin, and P. Pérez. Dense estimation of fluid flows. IEEE Trans. Pattern Anal. Machine Intell., 24(3):365-380, 2002.

[12] J. Fitzpatrick. The existence of geometrical density-image transformations corresponding to object motion. Comput. Vision, Graphics, Image Proc., 44(2):155-174, Nov. 1988.

[13] B. Galvin, B. McCane, K. Novins, D. Mason, and S. Mills. Recovering motion fields: an analysis of eight optical flow algorithms. In Proc. British Mach. Vis. Conf., Southampton, 1998.

[14] P. Héas, E. Mémin, D. Heitz, and P. Mininni. Bayesian selection of scaling laws for motion modeling in images. In International Conference on Computer Vision (ICCV'09), Kyoto, Japan, October 2009.

[15] D. Heitz, E. Mémin, and C. Schnoerr. Variational fluid flow measurements from image sequences: synopsis and perspectives. Exp. Fluids, 48(3):369-393, 2010.

[16] B. Horn and B. Schunck. Determining optical flow. Artificial Intelligence, 17:185-203, 1981.

[17] R. Kimmel and A. M. Bruckstein. Tracking level sets by level sets: a method for solving the shape from shading problem. Comput. Vis. Image Underst., 62(1):47-58, 1995.

[18] G. Le Besnerais and F. Champagnat. Dense optical flow by iterative local window registration. In Image Processing, 2005. ICIP 2005. IEEE International Conference on, volume 1, pages I - 137-40, sept. 2005.

[19] V. Lempitsky, S. Roth, and C. Rother. Fusionflow: Discrete-continuous optimization for optical flow estimation. In Proc. IEEE Comp. Vis. Patt. Rec. $(C V P R), 2008$.

[20] T. Lindeberg. Scale-space theory: A basic tool for analysing structures at different scales. J. of Applied Statistics, 21(2):224-270, 1994.

[21] B. Lucas and T. Kanade. An iterative image registration technique with an application to stereovision. In Int. Joint Conf. on Artificial Intel. (IJCAI), pages $674-679,1981$.

[22] A. Mansouri. Region tracking via level set PDEs without motion computation. IEEE Trans. Pattern Anal. Machine Intell., 24(7):947-961, 2003.

[23] E. Mémin and P. Pérez. Dense estimation and object-based segmentation of the optical flow with robust techniques. IEEE Trans. Image Processing, 7(5):703-719, 1998.

[24] H. Nagel. Extending the oriented smoothness constraint into the temporal domain and the estimation of derivatives of optical flow. In Proc. First European Conference On Comput. Vis., pages 139-148, Antibes, France, Apr 1990. Springer.

[25] P. Nesi. Variational approach to optical flow estimation managing discontinuities. Image and Vision Computing, 11(7):419-439, 1993.

[26] B. Oksendal. Stochastic differential equations. Spinger-Verlag, 1998.

[27] N. Papadakis, T. Corpetti, and E. Mémin. Dynamically consistent optical flow estimation. In IEEE Int. Conf. Comp. Vis.(ICCV’07), October 2007.

[28] N. Papadakis and E. Mémin. A variational technique for time consistent tracking of curves and motion. Journal of Mathematical Imaging and Vision, 31(1):81-103, May 2008

[29] N. Papenberg, A. Bruhn, T. Brox, S. Didas, and J. Weickert. Highly accurate optic flow computation with theoretically justified warping. Int. J. Comput. Vision, 67(2):141-158, 2006.

[30] N. Paragios and R. Deriche. Geodesic active regions: a new framework to deal with frame partition problems in computer vision. J. of Visual Communication and Image Representation, 13:249-268, 2002.

[31] B. Schunck. The image flow constraint equation. Comput. Vision, Graphics, Image Proc., 35:20-46, 1986.

[32] C. Tomasi and T. Kanade. Detection and tracking of points features. Technical report, School of Computer Science, Carnegie Mellon University, Pittsburgh, April 1991.

[33] O. Tretiak and L. Pastor. Velocity estimation from image sequences with second order differential operators. In Proc. 7th Int. Conf. On Pattern Recognition, pages 16-19, Montreal, 1984.

[34] J. Weber and J. Malik. Robust computation of optical flow in a multi-scale differential framework. Int. J. Comput. Vis., 14(1), 1995.

[35] A. Wedel, T. Pock, J. Braun, U. Franke, and D. Cremers. Duality tv-11 flow with fundamental matrix prior. In Image Vision and Computing, Auckland, New Zealand, November 2008.

[36] J. Weickert and C. Schnörr. Variational optic-flow computation with a spatio-temporal smoothness constraint. J. Math. Imaging and Vision, 14(3):245-255, 2001.

[37] Y. Wu, T. Kanade, C. Li, and J. Cohn. Image registration using wavelet-based motion model. Int. J. Comput. Vis., 38(2):129-152, 2000.

[38] L. Xu, J. Chen, and J. Jia. A segmentation based variational model for accurate optical flow estimation. In Eur. Conf. Comp. Vis., pages I: 671-684, 2008.

[39] J. Yuan, C. Schnörr, and E. Mémin. Discrete orthogonal decomposition and variational fluid flow estimation. Journ. of Mathematical Imaging and Vision, 28(1):67-80, 2007. 